\title{
CaNOP 3U CubeSat Attitude Determination and Control Testing System: Helmholtz Cage Design
}

\author{
Celestine Ananda and Nicholas Bartel \\ Carthage College, Kenosha WI
}

\begin{abstract}
Canopy Near-infrared Observing Project (CaNOP) is a WSGC-funded 3U CubeSat program to make multispectral reflectance measurements across a range of global forests. The project requires active attitude knowledge and control. This paper describes the design and construction of a test apparatus to validate the detumbling and attitude control systems of the CaNOP satellite. The apparatus generates a uniform 3-axis adjustable magnetic field to simulate on-orbit magnetic conditions. A Helmholtz cage implements six coils to create a region of uniform and adjustable magnetic field in which to test the orientation and attitude determination and control systems of a satellite.
\end{abstract}

\section{Introduction}

CubeSats are small satellites built by various institutions to conduct research in space at a fraction of the cost of a commercial satellite. Carthage's CubeSat team plans to design, build, and launch a 3 U CubeSat into space. In 2016, the Carthage CubeSat team was selected by NASA to design and build an earth-imaging satellite, named CaNOP (Canopy Near-infrared Observing Project), funded through the NASA Wisconsin Space Grant Consortium. The CubeSat will launch on ELaNA 23, a commercial resupply mission to the ISS from which it will then be ejected into space.

The technology goal of CaNOP is to demonstrate the functionality of a large hyperspectral imaging satellite (like those in the Landsat series of spacecraft) in the CubeSat form factor. The CaNOP science mission is to obtain medium-resolution images of global forest canopies with spectral resolution of $10 \mathrm{~nm}$ across the RGB and near-infra-red. This data will be used to compute spectral ratios such as NDVI to infer carbon content in both old-growth and harvested forests.

The CaNOP CubeSat needs to have precise control over the orientation of the satellite in order to capture clear images. The attitude control systems' actuators will correctly orient the CubeSat with data from the attitude determination systems' inertial and magnetic sensors. To ensure the attitude determination and control systems will function properly in space a uniform magnetic field must be replicated in a laboratory for testing purposes.

\section{Objective}

Our project's objective is to provide a test environment in which on-orbit conditions can be accurately reproduced so that the CaNOP CubeSat ADCS can be calibrated and its response to various tumbling conditions can be measured and validated.

\section{CaNOP ADCS Overview}

The attitude of a spacecraft is its rotational orientation in space relative to a defined reference coordinate system. Three parameters are required to define a rotational orientation (attitude) of a rigid body in a three-dimensional Euclidean space. The 3 
parameters required to define attitude can be defined qualitatively through the terms "yaw, pitch, and roll", as seen below [1].
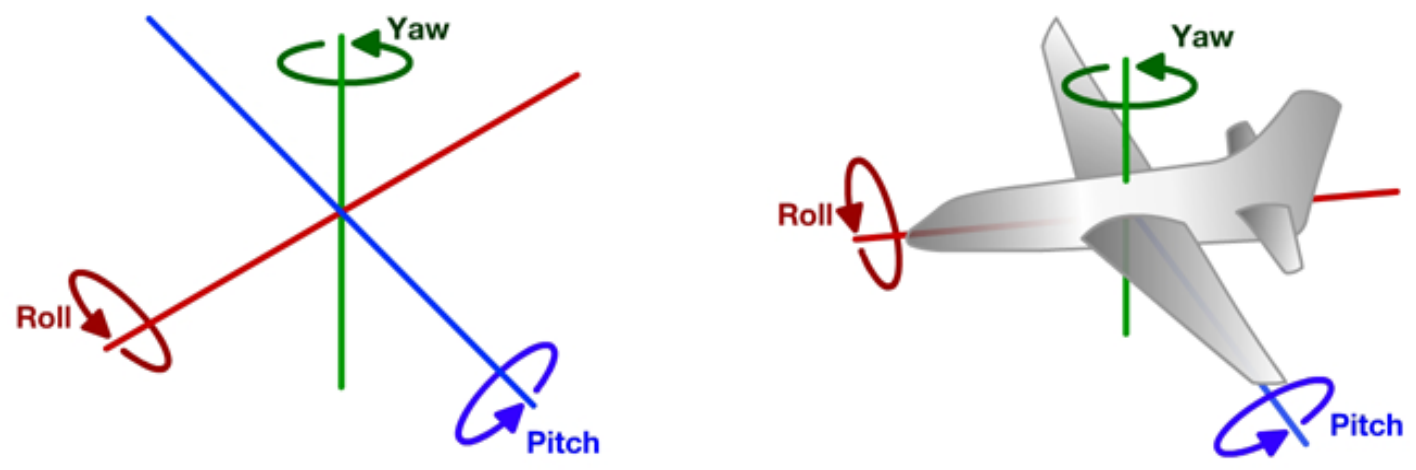

Fig. 1. Yaw, Pitch, and Roll

Three methods exist in defining these parameters quantitatively; a direction cosine matrix, Euler angles, and quaternions. The methods are used to parametrize a spacecraft's attitude with respect to a reference frame, determine attitude from one moment to the next using equations of motion, and to perform coordinate transformations. Quaternions are typically used as they are less computationally intense than the other methods and do not involve singularities.

The CaNOP CubeSat determines attitude to orient properly through inertial and magnetic sensors. This portion of the system is denoted as the "attitude determination system" (ADS). Three fine sun sensors determine the CubeSat's body angles with respect to the sun, two magnetometers detect the strength and orientation of the earth's magnetic field, and three MEMS (micro-electronic mechanical systems) gyroscopes, one per axis, determine the rate at which the satellite is tumbling.

Small satellites with attitude control capabilities typically include a set of three orthogonal magnetorquers. A magnetorquer is a set of electromagnetic coils that produce magnetic fields that supply a torque on the satellite as it aligns with the earth's field (with the aid of the magnetometers). To increase attitude control capabilities a set of three momentum wheels have been implemented to the CaNOP CubeSat. A reaction wheel is a small wheel that spins in the direction opposite of the satellite's spin to detumble through angular momentum conservation (with the aid of the inertial sensors). Additionally, the magnetorquers desaturate the reaction wheels as they cannot spin indefinitely. This portion of the system is denoted as the "attitude control system" (ACS).

\section{Helmholtz Cage Physics Principles}

A flat cylindrical coil of wire that creates a relatively uniform magnetic field in its interior has strength:

$$
B=\frac{N I \mu_{0}}{2 r}
$$

Where $B$ is the magnitude of the magnetic field, $\mu_{0}$ represents the permeability of free space (1.256610-6 $\mathrm{m} \mathrm{kg} \mathrm{s}^{2} \mathrm{~A}^{2}$ ), $I$ is the current in the wire, $r$ is the radius of the coil, and $N$ represents the number of loops in the wire. By aligning two identical current loops a more uniform 
magnetic field can be produced, known as Helmholtz coil pairs. Two coils of wire carrying a current $I$ that consist of $N$ loops produce a field of strength $B$. The magnetic field at the midpoint of two circular coils both of radius $r$ separated by a distance $r$ as seen in Fig. 2 [2] is calculated via:

$$
B=\frac{8}{5 \sqrt{5}} \frac{N I \mu_{0}}{r}
$$

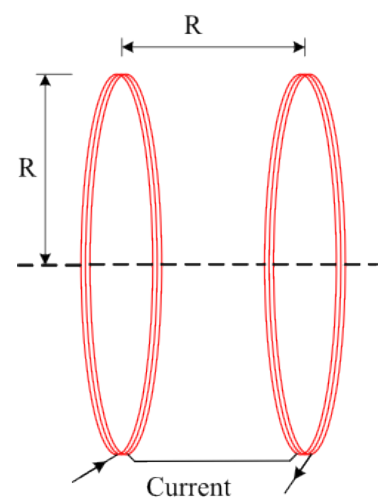

Fig 2. Helmholtz coil separation

Square coils can be used to increase the size of the field parallel to the coils [3]. The modified formula for the magnetic field at the midpoint between two square coils is defined as [4]:

$$
B=\frac{2 N I \mu_{0}}{\pi r} \frac{2}{\left(1+\gamma^{2}\right)\left(\sqrt{2+\gamma^{2}}\right)}
$$

Where $r$ represents half of a side length, and $\gamma$ represents the ratio between a full side length $(2 r)$ and the distance the coils are separated. The Helmholtz spacing is the coil separation for which the second derivative of the field vanishes at the center [5]. For a circular pair this spacing is the radii of the circles, for a square pair the spacing must be 0.5445 times the length of a full side [6]. Thus, $\gamma$ must equal 0.5445 . Actual coils will have rounded edges, but this error is negligible.

\section{Helmholtz Cage Requirements and Design}

The cage must be able to overcome the earth's magnetic field at our location, a laboratory at Carthage College $(.54 \mathrm{G})$, as well as simulate the magnetic field experienced in LEO (at maximum about $0.6 \mathrm{G}$ ), indicating that our cage must be able to produce fields of strengths at least $1.14 \mathrm{G}$ around each axis. We chose to overdesign to produce fields of strengths at least 2.28 G. To allow for testing of a $3 \mathrm{U}$ CubeSat the field must be uniform for a $34 \mathrm{~cm}^{3}$ testing volume.

To fulfill these requirements, we compared different gauges of wire with their respective maximum amperages due to the length-dependent resistance. We chose to use 60 turns per coil of 17 AWG magnet wire. Using Eqn. 3. with the parameters above for $1 \mathrm{~m} \times 1 \mathrm{~m}$ coils results in a uniform magnetic field of magnitude $2.33 \mathrm{G}$ over the region of interest. Fig. 3. illustrates the estimated peak strength and Fig. 4. illustrates the uniformity across the experimental volume [7]. The cage was designed using completely non-ferrous materials due to the potential effects on the fields produced. Aluminum 80/20 was chosen and used to build the support structure. An 
additional support stand made of aluminum and wood was incorporated to mount the satellite. The support stand contains a height adjustable turntable to allow field uniformity validation procedures and to allow the satellite to be positioned in the center of the field regardless of orientation. The coils are attached individually to 6 adjustable VDC power supplies in series with fuses and switches. Each coil required a different voltage due to the slight difference in resistance per coil. All fuses and switches terminate in a project box, as shown in Fig. 5.
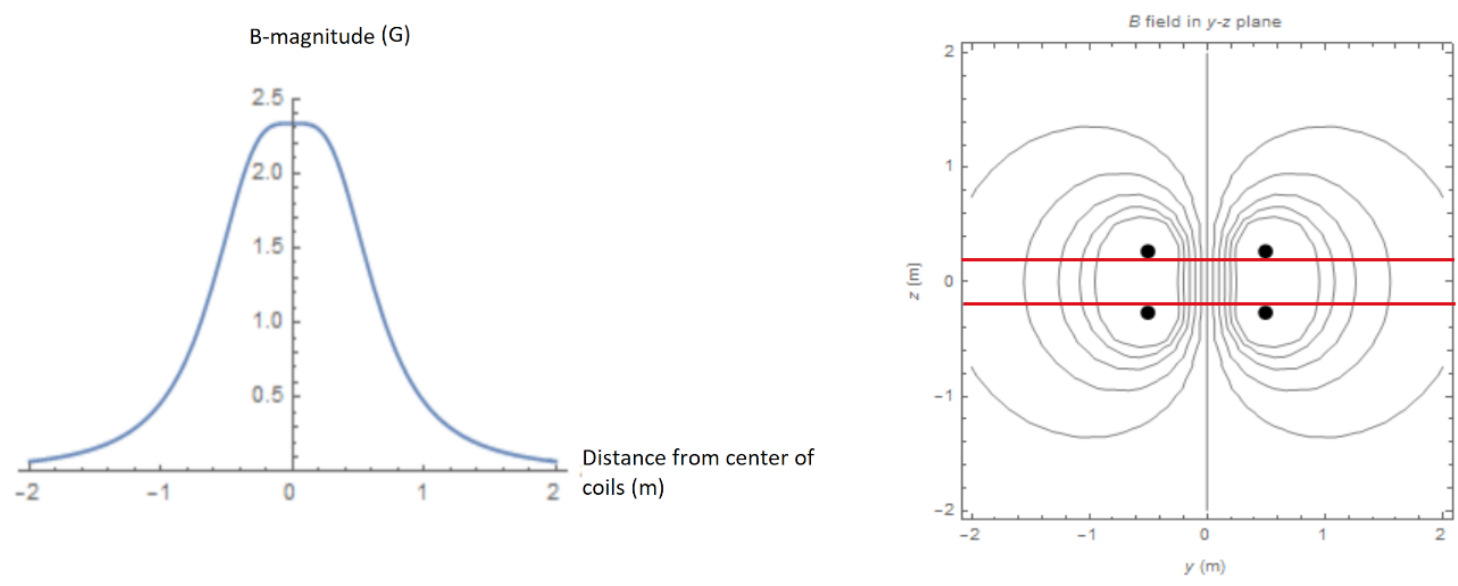

Fig 3. Estimated field strength peak

Fig. 4. Estimated region of field uniformity

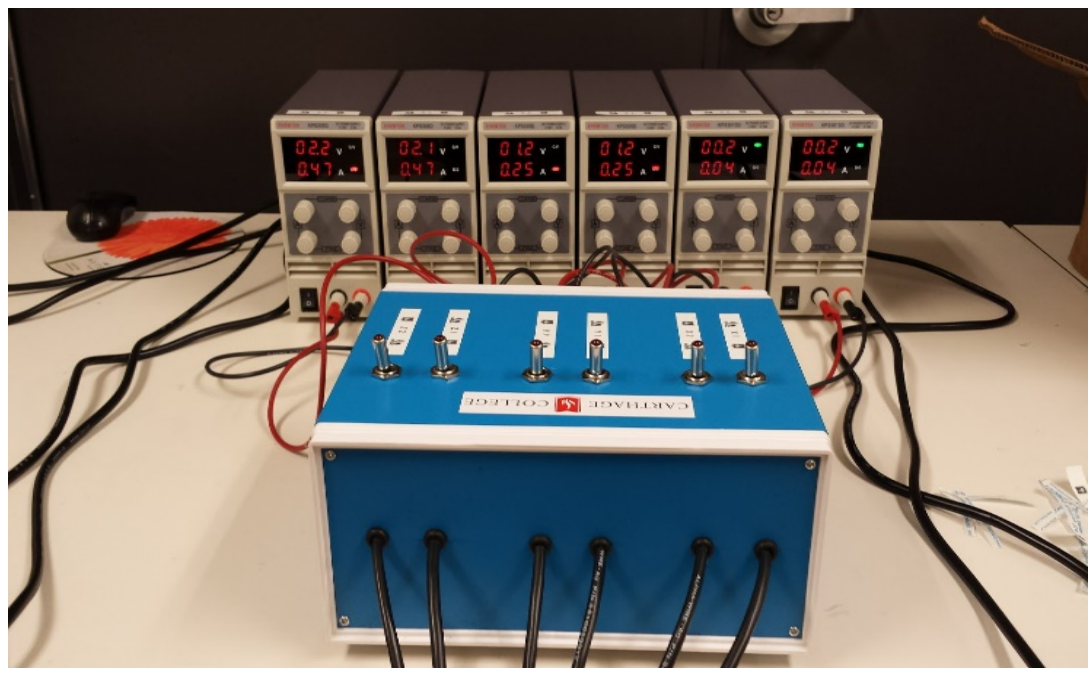

Fig. 5. All wires from coils are lead through a project box to individual power supplies

\section{Frame Design and Assembly}

The cage was designed using completely non-ferrous materials to eliminate unwanted induction fields. Aluminum 6061 was chosen and used to build the support structure. To avoid nesting frames and for simplicity, we built a $1 \mathrm{~m} \times 1 \mathrm{~m} \times 1 \mathrm{~m}$ cubical support structure and wrapped the wire around the cage instead of inlaying the wire in the supports themselves (as commonly seen in Helmholtz cage designs), Figures 6 and 7 show the final design and as-built cage. To easily accommodate wrapping the wire and in consideration of potentially ferrous metals in concrete, 
the vertical supports have an additional 10 in length to act as bases to provide shielding from any fields near the floor. To avoid using a separate support structure for the satellite during testing we incorporated a structure to attach to and fit inside the cage. This structure was constructed of aluminum $80 / 20$ as well as wood. The support stand contains a height adjustable turntable to give ease in field uniformity validation procedures and to allow the satellite to be positioned in the center of the field regardless of orientation. The 17 AWG wire was wrapped in rows of 10 by width and rows of 6 by height. A substantial number of zip ties bound the wires together tightly to ensure field uniformity.

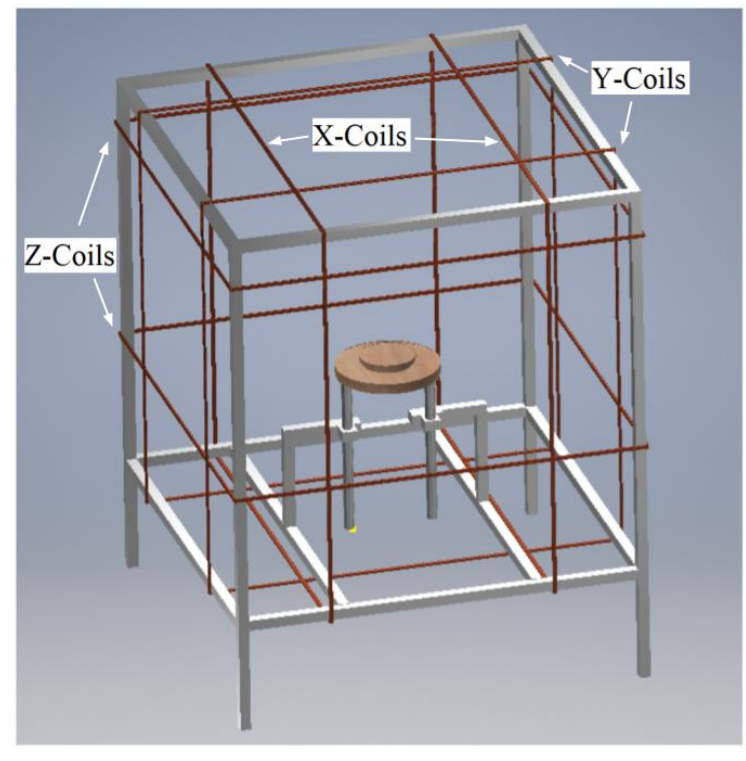

Fig. 6. CAD file image with axes labeled

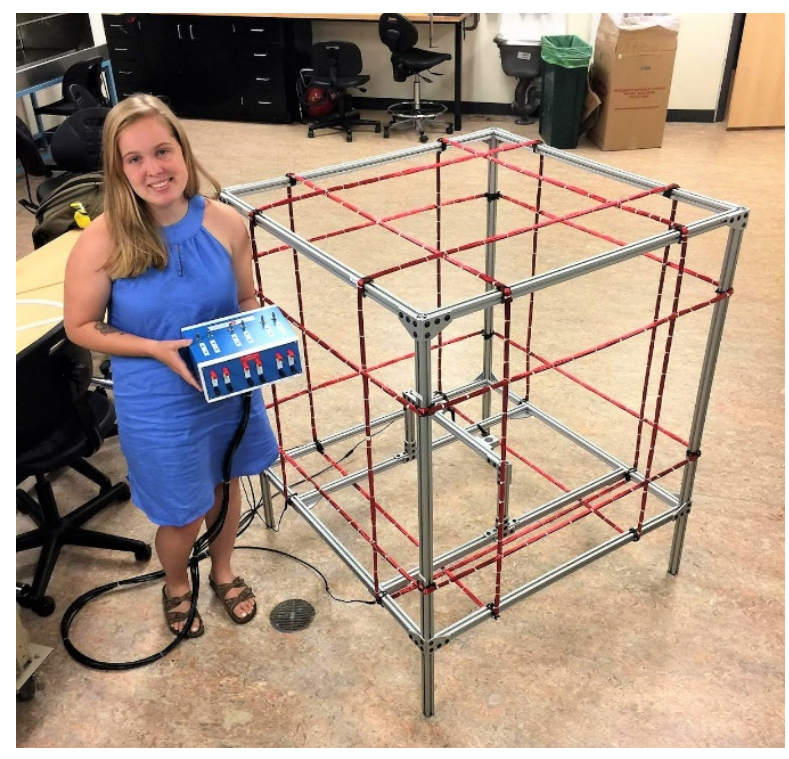

Fig. 7. Helmholtz cage without turntable

\section{Field Uniformity Validation Procedure}

The first step towards validating the magnetic fields' uniformity was to calibrate the cage through cancelling the earth's magnetic field. This was done by placing a magnetometer in the center of the turntable with the turntable in the center of the magnetic field. The corner locations of the cage were marked on the ground to ensure the testing area was consistent. We first took readings of the magnetic field before the cage was turned on at 64 separate grid locations marked on a piece of wood centered in the fields, each point $5 \mathrm{~cm} \times 5 \mathrm{~cm}$ apart. We then adjusted the applied voltages to each set of coils until the magnetometer read $0 \mathrm{G}$ in all axes. Initial readings were compared with the applied Helmholtz cage field readings and found that the cage did not deviate in uniformity by more than $0.05 \mathrm{G}$ in any direction in the region of uniform fields.

\section{CaNOP CubeSat Attitude Control Systems Testing Procedure}

A Nanoracks deployer will be releasing the CaNOP CubeSat into orbit from the International Space Station. This deployer holds multiple CubeSats and deploys them through a simple springloaded device. In this process a spin is applied to each satellite. Prior CubeSat missions have shown that an expected angular rate of deployment can be up to $10 \mathrm{deg} / \mathrm{s}$. The detumble function is meant to reduce the initial angular rate to a reasonable rate through the use of the 
magnetorquers so that the reaction wheels can be used for precise orientation maneuvers. The Helmholtz cage includes a height adjustable turntable with angles of 1-degree increments marked on the bottom outer edge. A uniform, non-dynamic magnetic field of $0.6 \mathrm{G}$ (akin to the geomagnetic field experienced on-orbit) will be generated by the Helmholtz Cage. The CubeSat will be placed on the turntable and given an initial angular velocity determined through video footage with zero actuation provided by the magnetorquers. We will measure the angular velocity of the CubeSat and the time that has passed. This process will then be repeated with active magnetorquers and sensors, the magnetorquers will produce a dipole moment to interact with the ambient magnetic field to produce a torque. The difference in the measured torques between the two cases should be equal to the torque applied by the magnetorquers. The torques are determined through measuring the angular accelerations and the moments of inertia of the CubeSat. These torques and time durations are then compared to expected values from simulations given the same initial angular velocity. The satellite is limited to one axis of rotation per test, with each test repeated for all three orientations.

\section{CaNOP CubeSat Attitude Determination Systems Calibration Procedure}

To offset any dipole bias the CubeSat creates the ADCS magnetometers must be calibrated before flight. Once the CubeSat has all components integrated we will force the CubeSat into a flight mode and spin the system on a turntable inside a Helmholtz cage. We will log the raw magnetometer data as frequently as possible in as many orientations as possible. The logged data shall be passed to Clyde Space to calibrate the data. Clyde Space will then send an updated configuration file to install to the ADCS Motherboard via the I2C Interface, as well as all other components the Motherboard is integrated into. Other CubeSat missions commonly affix magnets on the satellite to offset any magnetic biases, the CaNOP CubeSat eliminates this step through the vendor's calibration. Additionally, each sun sensor will be tested using a sun simulator with collimated light the intensity of the sun. Each sensor will be illuminated separately while monitoring outputs from the FSS and CSS memory locations to ensure each sensor is functioning properly.

\section{Helmholtz Cage Results and Summary}

Preliminary testing shows the fields produced deviate by less than $0.05 \mathrm{G}$ within the $34 \mathrm{~cm}^{3}$ volume. It was found that with a current of $2.5 \mathrm{~A}$ the X-Coils can produce B-fields of strengths $2.53 \mathrm{G}$, the Y-Coils of strengths $2.62 \mathrm{G}$, and the Z-Coils of strengths $2.45 \mathrm{G}$ - exceeding calculated and approximated expectations. We have designed, constructed, and demonstrated the operation of a low-cost, high precision Helmholtz Cage for the calibration and testing of $3 \mathrm{U}$ CubeSats.

\section{Acknowledgement}

We would like to thank the NASA Wisconsin Space Grant Consortium, and the NASA Undergraduate Student Instrumentation Program (USIP). Work supported by NASA USIP Award \#NNX16A170A. Further, we would like to thank our mentor, Dr. Crosby, for his time and dedication to the CaNOP CubeSat project.

\section{Citations}

[1] "Pitch, Roll, and Yaw." Touring Machine Company, Touring Machine Company, 29

Nov. 2007, www.touringmachine.com/Articles/aircraft/6/. 
[2] "Helmholtz Coil." Helmholtz Coil, www.accelinstruments.com/HelmholtzCoil/Helmholtz-Coil.html.

[3] Pramoda, K. e. a., "Square Loop Coil System for Balancing and Calibration of SecondOrder SQUID Gradiometers," Journal of Physics: Conference Series 208, 2010

[4] Brewer, Megan R. "CubeSat Attitude Determination and Helmholtz Cage Design." DEPARTMENT OF THE AIR FORCE AIR UNIVERSITY, 2012.

[5] Rudd, M. E., and J. R. Craig. "Optimum Spacing of Square and Circular Coil Pairs." Review of Scientific Instruments, vol. 39, no. 9, 1968, pp. 1372-1374., doi:10.1063/1.1683678.

[6] Lee, G., Kang, C., and Chang, J., "Square Loop Coil System for Balancing and Calibration of Second-Order SQUID Gradiometers, 2011 IEEE," IEEE Transactions on Applied Superconductivity, Vol. 17, No. 2, 2007, pp. 3769-377

[7] Euripides, Peter. "Square Helmholtz Coils CDF." Wolfram Demonstrations Project, Peter Euripides, 27 Dec. 2007, demonstrations.wolfram.com/SquareHelmholtzCoils/. 